\title{
A REGULARITY UPGRADE OF PRESSURE
}

\author{
DONG LI AND XIAOYI ZHANG
}

\begin{abstract}
For the incompressible Euler equations the pressure formally scales as a quadratic function of velocity. We provide several optimal regularity estimates on the pressure by using regularity of velocity in various Sobolev, Besov and Hardy spaces. Our proof exploits the incompressibility condition in an essential way and is deeply connected with the classic Div-Curl lemma which we also generalise as a fractional Leibniz rule in Hardy spaces. To showcase the sharpness of results, we construct a class of counterexamples at several end-points.
\end{abstract}

\section{INTRODUCTION}

The $n$-dimensional $(n \geq 2)$ incompressible Euler equation takes the form

$$
\left\{\begin{array}{l}
\partial_{t} u+(u \cdot \nabla) u=-\nabla p, \quad(t, x) \in(0, \infty) \times \mathbb{R}^{n}, \\
\nabla \cdot u=0, \\
\left.u\right|_{t=0}=u_{0},
\end{array}\right.
$$

where $u:[0, \infty) \times \mathbb{R}^{n} \rightarrow \mathbb{R}^{n}, p:[0, \infty) \times \mathbb{R}^{n} \rightarrow \mathbb{R}$ represent velocity and pressure of the underlying fluid respectively. In this work we shall not consider the Cauchy problem or wellposedness issues at all. Instead we regard $u$ as a given solution to (1.1) in appropriate function spaces. Our main objective is to study the regularity properties of the pressure $p$ in terms of the known velocity field $u$. By taking the divergence on both sides of the first equation in (1.1), we get

$$
-\Delta p=\nabla \cdot((u \cdot \nabla) u) \text {. }
$$

Equation (1.2) will be our main object of study. To simplify the discussion we shall completely ignore the explicit time dependence and only focus on spatial regularity. Regarding (1.2) as a Poisson problem for the pressure $p$, it is well known that $p$ is determined up to a harmonic part. This degree of freedom can be eliminated by supplying some decay conditions at spatial infinity. Alternatively to simplify matters, in the following discussion, we shall take the convention that $p$ is identified with the expression

$$
(-\Delta)^{-1} \nabla \nabla(u \otimes u)=\sum_{l, k}(-\Delta)^{-1} \partial_{l} \partial_{k}\left(u_{l} u_{k}\right)
$$

which is well-defined by putting suitable assumptions on the velocity vector $u$ (e.g. $u$ is Schwartz). Our main focus is the quantitative estimates of $p$ in terms of $u$ in various functional spaces.

Note that since $u$ is divergence-free, one can rewrite (1.2) as

$$
-\Delta p=\sum_{k, l} \partial_{k} \partial_{l}\left(u_{k} u_{l}\right)
$$


Alternatively, we can rewrite (1.3) as

$$
-\Delta p=\sum_{k, l}\left(\partial_{k} u_{l}\right)\left(\partial_{l} u_{k}\right)
$$

There are some subtle differences between the expressions (1.2)-(1.4). Assume that $u \in W^{1, q}\left(\mathbb{R}^{n}\right)$ for some $n<q<\infty$. If we only make use of (1.2), then

$$
\nabla p=(-\Delta)^{-1} \nabla \nabla \cdot(u \cdot \nabla u),
$$

which gives $\nabla p \in L^{q}\left(\mathbb{R}^{n}\right)$. By using (1.3) it is easy to check that $p \in L^{q}\left(\mathbb{R}^{n}\right)$. Thus we obtain $p \in W^{1, q}\left(\mathbb{R}^{n}\right)$. On the other hand, if we use (1.4), then clearly $p \in W^{2, \frac{q}{2}}\left(\mathbb{R}^{n}\right)$. This is apparently a better estimate in view of the embedding $W^{2, \frac{q}{2}}\left(\mathbb{R}^{n}\right) \hookrightarrow W^{1, q}\left(\mathbb{R}^{n}\right)$. As it turns out, this "upgrade of regularity" phenomenon is quite generic. For example it can be generalised to Sobolev spaces $W^{s, q}$ with $0<s \leq 1,2<q<\infty$ and even Besov spaces. We have the following theorem.

Theorem 1.1 (Sobolev and Besov). Suppose $u \in \mathcal{S}\left(\mathbb{R}^{n}\right)$. Then for $0 \leq s \leq 1$ and $1<q<\infty$, we have

$$
\|p\|_{W^{2 s, q}\left(\mathbb{R}^{n}\right)} \lesssim\|u\|_{W^{s, 2 q}\left(\mathbb{R}^{n}\right)}^{2} .
$$

If $1 \leq q, r \leq \infty$ and $0<s<1$, then

$$
\|p\|_{\dot{B}_{q, r}^{2 s}\left(\mathbb{R}^{n}\right)} \lesssim\|u\|_{\dot{B}_{2 q, 2 r}^{s}\left(\mathbb{R}^{n}\right)}^{2}
$$

In particular for Hölder spaces we have for $0<s<1$,

$$
\|p\|_{\dot{B}_{\infty, \infty}^{2 s}} \lesssim\|u\|_{\dot{B}_{\infty, \infty}^{s}}^{2} .
$$

On the other hand, for $s=1$ the corresponding Besov estimate does not hold and can be replaced by

$$
\|p\|_{\dot{B}_{\infty, \infty}^{2}} \lesssim\|\nabla u\|_{\infty}^{2}
$$

Remark. (1.8) is a simple consequence of (1.4) and thus we omit the proof.

Remark. For Schwartz functions $f: \mathbb{R}^{n} \rightarrow \mathbb{R}^{n}, g: \mathbb{R}^{n} \rightarrow \mathbb{R}^{n}$ with the property $\nabla \cdot f=\nabla \cdot g=0$, one can consider the bilinear operator

$$
B(f, g)=\sum_{l, k=1}^{n} \partial_{l} \partial_{k} \Delta^{-1}\left(f_{l} g_{k}\right)
$$

Same proof as in Theorem 1.1 yields that for $0 \leq s \leq 1$ and $1<q<\infty$,

$$
\|B(f, g)\|_{W^{2 s, q}} \lesssim\|f\|_{W^{s, 2 q}}\|g\|_{W^{s, 2 q}} ;
$$

and for $0<s<1,1 \leq q, r \leq \infty$,

$$
\|B(f, g)\|_{\dot{B}_{q, r}^{2 s}} \lesssim\|f\|_{\dot{B}_{2 q, 2 r}^{s}}\|g\|_{\dot{B}_{2 q, 2 r}^{s}} .
$$

Remark. The estimate (1.7) shows that for $0<s<\frac{1}{2}$,

$$
\|p\|_{\dot{C}^{2 s}} \lesssim\|u\|_{\dot{C}^{s}}^{2}
$$

and for $\frac{1}{2}<s<1$,

$$
\|\nabla p\|_{\dot{C}^{2 s-1}} \lesssim\|u\|_{\dot{C}^{s}}^{2}
$$

One should recall that $\|f\|_{\dot{C}^{s}} \sim\|f\|_{\dot{B}_{\infty, \infty}^{s}}$ for $0<s<1$. Thus the Besov formulation connects these two estimates in a most natural way. 
Note from (1.3) it is evident that $p=\mathcal{R}\left(O\left(u^{2}\right)\right)$ where $\mathcal{R}$ is a Riesz-type operator. The usual product rule in Hölder spaces says that if $f, g \in C^{s}$, then $f g \in C^{s}$. Thus by (1.3) one should only expect $p \in C^{s}$ if $u \in C^{s}$. However here by using Theorem 1.1. one can prove that $p \in \dot{B}_{\infty, \infty}^{2 s}$ as long as $0<s<1$. Roughly speaking, we are asserting that the $2 s$-derivative on $p$ can fall "evenly" into each composing velocity:

$$
|\nabla|^{2 s} p \sim \mathcal{R}\left(\left.|\nabla|\right|^{s} u|\nabla|{ }^{s} u\right)
$$

and there do not appear terms such as $O\left(|\nabla|^{s-} u|\nabla|^{s+} u\right)$.

There exists an analogue of Theorem 1.1 in Hardy space. The following theorem can be regarded as the case $q=2$ in Theorem 1.1

Theorem 1.2 (Hardy space). Let $0<s \leq 1$. Then for $u \in \mathcal{S}\left(\mathbb{R}^{n}\right)$,

$$
\left\||\nabla|^{2 s} p\right\|_{\mathcal{H}^{1}\left(\mathbb{R}^{n}\right)} \lesssim\|u\|_{W^{s, 2}\left(\mathbb{R}^{n}\right)}^{2} .
$$

In the case $s=1$, the operator can be replaced by a general second order derivative $\partial^{2}=\partial_{i} \partial_{j}$ for any $i, j \in\{1, \cdots, n\}$.

Remark. For $0<s<1$ one has the stronger estimate $p \in \dot{B}_{1,1}^{2 s}$ thanks to theorem 1.1 .

In the proof of Theorem 1.2 we need to exploit the incompressibility of velocity which provides cancelation of some high frequency interaction terms in the nonlinearity. As it turns out, in the 3D case, this is deeply linked to the "Div-Curl" lemma in Coifman-Lions-Meyer-Semmes [1]. In its simplest formulation, the DivCurl lemma asserts that if $\nabla \cdot f=\nabla \times g=0$, then

$$
\|f \cdot g\|_{\mathcal{H}^{1}} \lesssim\|f\|_{p}\|g\|_{p^{\prime}}
$$

where $1<p<\infty$ and $p^{\prime}=p /(p-1)$. In light of the proof in Theorem 1.2, we can obtain the following generalisation which can also be regarded as a fractional Leibniz rule in Hardy space.

Theorem 1.3 (Generalised Div-Curl lemma). Let $1<p_{1}, p_{2}<\infty$ and $-1<s<$ $\infty$. Then for any Schwartz $f, g: \mathbb{R}^{3} \rightarrow \mathbb{R}^{3}$ with

$$
\nabla \cdot f=0, \quad \nabla \times g=0,
$$

we have

$$
\left\||\nabla|^{s}(f \cdot g)\right\|_{\mathcal{H}^{1}} \lesssim\left\||\nabla|^{s} f\right\|_{p_{1}}\|g\|_{p_{1}^{\prime}}+\|f\|_{p_{2}^{\prime}}\left\||\nabla|^{s} g\right\|_{p_{2}}
$$

whenever the RHS is finite. When $-1<s \leq 0$, we have

$$
\left\||\nabla|^{s}(f \cdot g)\right\|_{\mathcal{H}^{1}} \lesssim \min \left\{\left\||\nabla|^{s} f\right\|_{p_{1}}\|g\|_{p_{1}^{\prime}},\|f\|_{p_{2}^{\prime}}\left\||\nabla|^{s} g\right\|_{p_{2}}\right\} .
$$

Remark. For $s>0$ one does not need the "Div-Curl" condition to derive the estimate. The only nontrivial case is $-1<s \leq 0$.

In Theorem 1.1. for the Besov case we only considered the regime $0<s<1$ and left out the cases $s=0$ and $s=1$. It is instructive to investigate these end-point cases. As it turns out, the corresponding regularity estimate for pressure fails in general. To clarify this point we construct counterexamples.

Theorem 1.4. The estimate (1.7) fails in the case $s=0,1$. More specifically, for any $\varepsilon>0$, there exists divergence free $u \in \mathcal{S}\left(\mathbb{R}^{n}\right)$ for with

$$
\|u\|_{\dot{B}_{\infty, \infty}^{1}}+\|u\|_{L^{2}} \leq 1
$$


but

$$
\|p\|_{\dot{B}_{\infty, \infty}^{2}}>\frac{1}{\varepsilon}
$$

A similar statement holds for $s=0$. Here $\mathcal{S}\left(\mathbb{R}^{n}\right)$ is the class of Schwartz functions.

Remark. Our construction shows that the inflation occurs at high frequencies.

In the estimate (1.7), when $s=\frac{1}{2}, p \in \dot{B}_{\infty, \infty}^{1}$. The norm of $\dot{B}_{\infty, \infty}^{1}$ is much weaker than the norm of $C^{1}$. Of course it is not difficult to construct a function which lies in $\dot{B}_{\infty, \infty}^{1}$ but not in $C^{1}$. However, it is not obvious to construct a $p \notin C^{1}$ starting from a divergence free $u \in C^{\frac{1}{2}}$. The following theorem gives such a result.

Theorem 1.5. There exists $u \in C^{\frac{1}{2}} \cap L^{2}$ for which $p \notin C^{1}$.

The last result says the estimate (1.9) fails at the endpoint case $s=0$.

Proposition 1.6. There exists divergence-free $u \in \mathcal{S}\left(\mathbb{R}^{n}\right)$ such that $p \notin L^{1}\left(\mathbb{R}^{n}\right)$.

We should remark that the case of domains with appropriate boundary conditions can be explored further and we plan to address it elsewhere.

The rest of this paper is organised as follows. In Section 2 we introduce some basic notation and collect some preliminary estimates. In Section 3 we give the regularity estimates of pressure in aforementioned function spaces. In Section 4 we give the construction of counterexamples at various end-point cases.

Acknowledgements. D. Li was supported in part by a start-up grant from HKUST and HK RGC grant 16307317. X. Zhang was supported by Simons Collaboration grant.

\section{PRELIMINARIES}

For any real number $a \in \mathbb{R}$, we denote by $a+$ the quantity $a+\epsilon$ for sufficiently small $\epsilon>0$. The numerical value of $\epsilon$ is unimportant and the needed smallness of $\epsilon$ is usually clear from the context. The notation $a$ - is similarly defined.

For any two quantities $X$ and $Y$, we denote $X \lesssim Y$ if $X \leq C Y$ for some constant $C>0$. Similarly $X \gtrsim Y$ if $X \geq C Y$ for some $C>0$. We denote $X \sim Y$ if $X \lesssim Y$ and $Y \lesssim X$. The dependence of the constant $C$ on other parameters or constants are usually clear from the context and we will often suppress this dependence. We shall denote $X \lesssim_{Z_{1}, Z_{2}, \cdots, Z_{k}} Y$ if $X \leq C Y$ and the constant $C$ depends on the quantities $Z_{1}, \cdots, Z_{k}$.

We denote by $\mathcal{S}\left(\mathbb{R}^{n}\right)$ the space of Schwartz functions and $\mathcal{S}^{\prime}\left(\mathbb{R}^{n}\right)$ the space of tempered distributions. For any function $f: \mathbb{R}^{n} \rightarrow \mathbb{R}$, we use $\|f\|_{L^{q}\left(\mathbb{R}^{n}\right)},\|f\|_{L^{q}}$ or sometimes $\|f\|_{q}$ to denote the usual Lebesgue $L^{q}$ norm for $0<q \leq \infty$. For $s>0$, $1<q<\infty$, we recall the Sobolev norms

$$
\|f\|_{\dot{W}^{s, q}\left(\mathbb{R}^{n}\right)}=\left\||\nabla|^{s} f\right\|_{q}, \quad\|f\|_{W^{s, q}\left(\mathbb{R}^{n}\right)}=\|f\|_{q}+\left\||\nabla|^{s} f\right\|_{q} .
$$

For a sequence of real numbers $\left(a_{j}\right)_{j=-\infty}^{\infty}$, we denote

$$
\left(a_{j}\right)_{l_{j}^{q}}=\left\|\left(a_{j}\right)_{j \in \mathbb{Z}}\right\|_{l^{q}}=\left\{\begin{array}{l}
\left(\sum_{j \in \mathbb{Z}}\left|a_{j}\right|^{q}\right)^{\frac{1}{q}}, \quad \text { if } 0<q<\infty, \\
\sup _{j}\left|a_{j}\right|, \quad \text { if } q=\infty .
\end{array}\right.
$$


We shall often use mixed-norm notation. For example, for a sequence of functions $f_{j}: \mathbb{R}^{n} \rightarrow \mathbb{R}$, we will denote (below $0<r<\infty$ )

$$
\left\|\left(f_{j}\right)_{l_{j}^{r}}\right\|_{q}=\left\|\left(\sum_{j}\left|f_{j}(x)\right|^{r}\right)^{\frac{1}{r}}\right\|_{L_{x}^{q}\left(\mathbb{R}^{n}\right)},
$$

with obvious modification for $q=\infty$.

We use the following convention for the Fourier transform:

$$
(\mathcal{F} f)(\xi)=\hat{f}(\xi)=\int_{\mathbb{R}^{n}} e^{-i x \cdot \xi} f(x) d x .
$$

$\mathcal{F}^{-1}$ is the inverse Fourier transform:

$$
\mathcal{F}^{-1} g(x)=\frac{1}{(2 \pi)^{n}} \int_{\mathbb{R}^{n}} e^{i x \cdot \xi} g(\xi) d \xi .
$$

For $s \in \mathbb{R}$, the fractional Laplacian $|\nabla|^{s}=(-\Delta)^{s / 2}$ corresponds to the multiplier $|\xi|^{s}$ on the Fourier side (whenever it is well-defined). Sometimes we also denote $|\nabla|^{s}$ as $D^{s}$.

We first introduce the Littlewood-Paley operators.

Let $\tilde{\phi} \in C_{c}^{\infty}\left(\mathbb{R}^{n}\right)$ be such that

$$
\tilde{\phi}(\xi)= \begin{cases}1, & |\xi| \leq 1 \\ 0, & |\xi|>\frac{7}{6}\end{cases}
$$

Let $\phi_{c}(\xi)=\tilde{\phi}(\xi)-\tilde{\phi}(2 \xi)$ which is supported on $\frac{1}{2} \leq|\xi| \leq \frac{7}{6}$. For any $f \in \mathcal{S}\left(\mathbb{R}^{n}\right)$, $j \in \mathbb{Z}$, define

$$
\begin{aligned}
& \mathcal{F}\left(P_{\leq j} f\right)(\xi)=\widehat{P_{\leq j} f}(\xi)=\tilde{\phi}\left(2^{-j} \xi\right) \hat{f}(\xi), \\
& \widehat{P_{j} f}(\xi)=\phi_{c}\left(2^{-j} \xi\right) \hat{f}(\xi), \quad \xi \in \mathbb{R}^{n} .
\end{aligned}
$$

We will denote $P_{>j}=I-P_{\leq j}$ ( $I$ is the identity operator). Sometimes for simplicity of notation (and when there is no obvious confusion) we will write $f_{j}=P_{j} f$, $f_{\leq j}=P_{\leq j} f$ and $f_{a \leq . \leq b}=\sum_{a \leq j \leq b} f_{j}$. By using the support property of $\phi$, we have $P_{j} P_{j^{\prime}}=0$ whenever $\left|j-j^{\prime}\right|>1$. This property will be useful in product decompositions. For example the Bony paraproduct for a pair of functions $f, g$ take the form

$$
f g=\sum_{i \in \mathbb{Z}} f_{i} \tilde{g}_{i}+\sum_{i \in \mathbb{Z}} f_{i} g_{\leq i-2}+\sum_{i \in \mathbb{Z}} g_{i} f_{\leq i-2}
$$

where $\tilde{g}_{i}=g_{i-1}+g_{i}+g_{i+1}$.

The fattened operators $\tilde{P}_{j}$ are defined by

$$
\tilde{P}_{j}=\sum_{l=-n_{1}}^{n_{2}} P_{j+l},
$$

where $n_{1} \geq 0, n_{2} \geq 0$ are some finite integers whose values play no role in the argument.

We will often use the following Bernstein inequalities without explicit mentioning. 
Proposition 2.1 (Bernstein inequality). Let $1 \leq p \leq q \leq \infty$. For any $f \in L^{p}\left(\mathbb{R}^{n}\right)$, $j \in \mathbb{Z}$, we have

$$
\begin{aligned}
& \left\|P_{\leq j} f\right\|_{q}+\left\|P_{j} f\right\|_{q} \lesssim 2^{j n\left(\frac{1}{p}-\frac{1}{q}\right)}\|f\|_{p} ; \\
& \left\||\nabla|^{s} P_{\leq j} f\right\|_{p} \lesssim 2^{j s}\|f\|_{p}, \quad \forall s \geq 0 ; \\
& \left\|P_{j} f\right\|_{p} \sim 2^{-j s}\left\||\nabla|^{s} P_{j} f\right\|_{p}, \quad \forall s \in \mathbb{R} ; \\
& \left\|P_{>j} f\right\|_{p} \lesssim 2^{-j s}\left\||\nabla|^{s} f\right\|_{p}, \quad \forall s \geq 0 .
\end{aligned}
$$

For $s \in \mathbb{R}, 1 \leq p, q \leq \infty$, the homogeneous Besov $\dot{B}_{p, q}^{s}$ (semi)-norm is given by

$$
\|f\|_{\dot{B}_{p, q}^{s}\left(\mathbb{R}^{n}\right)}=\left\|\left(2^{j s}\left\|f_{j}\right\|_{p}\right)\right\|_{l_{j}^{q}}=\left\{\begin{array}{l}
\left(\sum_{j \in \mathbb{Z}} 2^{j q s}\left\|P_{j} f\right\|_{L_{x}^{p}\left(\mathbb{R}^{n}\right)}^{q}\right)^{\frac{1}{q}}, \quad \text { if } 1 \leq q<\infty ; \\
\sup _{j \in \mathbb{Z}} 2^{j s}\left\|P_{j} f\right\|_{L_{x}^{p}\left(\mathbb{R}^{n}\right)}, \quad \text { if } q=\infty .
\end{array}\right.
$$

For $s \in \mathbb{R}, 1 \leq p, q \leq \infty$,

$$
\|f\|_{B_{p, q}^{s}\left(\mathbb{R}^{n}\right)}=\left\|P_{\leq 0} f\right\|_{p}+\left\|\left(2^{j s}\left\|f_{j}\right\|_{p}\right)\right\|_{l_{j}^{q}(j \geq 1)} .
$$

For $s>0$ and $1 \leq p, q \leq \infty$, it is easy to check that

$$
\|f\|_{B_{p, q}^{s}} \sim\|f\|_{p}+\|f\|_{\dot{B}_{p, q}^{s}} .
$$

Proposition 2.2 (Continuity in "s"). Let $1 \leq p, q \leq \infty$. Suppose $\|f\|_{B_{p, q}^{s}}<\infty$, then

$$
\lim _{\substack{\tilde{s} \rightarrow s \\ \tilde{s}<s}}\|f\|_{B_{p, q}^{\tilde{s}}}=\|f\|_{B_{p, q}^{s}} .
$$

On the other hand, if for some $\epsilon_{0}>0$,

$$
\sup _{\tilde{s} \in\left(s-\epsilon_{0}, s\right)}\|f\|_{B_{p, q}^{\tilde{s}}}<\infty,
$$

then $\|f\|_{B_{p, q}^{s}}<\infty$, and

$$
\lim _{\substack{\tilde{s} \rightarrow s \\ s-\epsilon_{0}<\tilde{s}<s}}\|f\|_{B_{p, q}^{\tilde{s}}}=\|f\|_{B_{p, q}^{s}} .
$$

If for some $\epsilon_{1}>0,\|f\|_{B_{p, q}^{s+\epsilon_{1}}}<\infty$, then

$$
\lim _{\substack{\tilde{s} \rightarrow s \\ \tilde{s}>s}}\|f\|_{B_{p, q}^{\tilde{s}}}=\|f\|_{B_{p, q}^{s}} .
$$

Remark. This simple proposition explains the folklore fact that the limit $\alpha \rightarrow 1$ for Hölder $C^{\alpha}, 0<\alpha<1$, is $B_{\infty, \infty}^{1}$; and the limit $\alpha \rightarrow 0$ for Hölder $C^{1, \alpha}, 0<\alpha<1$ is $B_{\infty, \infty}^{1}$.

Proof. If $q<\infty$, one can just use Monotone Convergence to obtain

$$
\lim _{\substack{s \rightarrow s \\ \tilde{s}<s}}\left(2^{j \tilde{s}}\left\|P_{j} f\right\|_{p}\right)_{l_{j}^{q}(j \geq 1)}=\left(2^{j s}\left\|P_{j} f\right\|_{p}\right)_{l_{j}^{q}(j \geq 1)} .
$$

Also for $q<\infty$, by using Dominated convergence, we have

$$
\lim _{\substack{\tilde{s} \rightarrow s \\ s<\tilde{s}<s+\epsilon_{1}}}\left(2^{j \tilde{s}}\left\|P_{j} f\right\|_{p}\right)_{l_{j}^{q}(j \geq 1)}=\left(2^{j s}\left\|P_{j} f\right\|_{p}\right)_{l_{j}^{q}(j \geq 1)} .
$$


If $q=\infty$, one observes that

$$
\begin{aligned}
& \sup _{\tilde{s}<s} \sup _{j \geq 1} 2^{j \tilde{s}}\left\|P_{j} f\right\|_{p}=\sup _{j \geq 1} 2^{j s}\left\|P_{j} f\right\|_{p} ; \\
& \lim _{\substack{\tilde{s} \rightarrow s \\
s<\tilde{s}<s+\epsilon_{1}}} \sup _{j \geq 1} 2^{j \tilde{s}}\left\|P_{j} f\right\|_{p}=\sup _{j \geq 1} 2^{j s}\left\|P_{j} f\right\|_{p} .
\end{aligned}
$$

We will use the following characterisation for the $\dot{C}^{\alpha}$ space. Recall that for $0<\alpha<1$,

$$
\|f\|_{\dot{C}^{\alpha}}:=\sup _{x \neq y} \frac{|f(x)-f(y)|}{|x-y|^{\alpha}}
$$

and

$$
\|f\|_{C^{\alpha}}=\|f\|_{\infty}+\|f\|_{\dot{C}^{\alpha}} .
$$

Lemma 2.3. Let $0<\alpha<1$. Then for any $f$ with $\|f\|_{\dot{C}^{\alpha}\left(\mathbb{R}^{n}\right)}<\infty$, we have

$$
\|f\|_{\dot{C}^{\alpha}} \sim\|f\|_{\dot{B}_{\infty, \infty}^{\alpha}} .
$$

Remark. Similarly for $\alpha=1$ and $f \in \mathcal{S}\left(\mathbb{R}^{n}\right)$, one has the equivalence

$$
\sup _{h \neq 0, x \in \mathbb{R}^{n}} \frac{|f(x+h)+f(x-h)-2 f(x)|}{|h|} \sim\|f\|_{\dot{B}_{\infty, \infty}^{1}} .
$$

Remark. It follows easily that for $0<\alpha<1$, and any $f \in \mathcal{S}\left(\mathbb{R}^{n}\right)$, we have

$$
\|\nabla f\|_{\dot{C}^{\alpha}} \sim\|\nabla f\|_{\dot{B}_{\infty, \infty}^{\alpha}} \sim\|f\|_{\dot{B}_{\infty, \infty}^{1+\alpha}} .
$$

Proof. Easy to check that $\|f\|_{\dot{B}_{\infty, \infty}^{\alpha}} \lesssim\|f\|_{\dot{C}^{\alpha}}$. To show the reverse inequality, one can fix $x \neq y$ and note that with the assumption $\|f\|_{\dot{C}^{\alpha}}<\infty$ one has

$$
\lim _{J \rightarrow \infty}\left|\left(P_{\leq-J} f\right)(x)-\left(P_{\leq-J} f\right)(y)\right| \lesssim \lim _{J \rightarrow \infty}\left\|\nabla P_{\leq-J} f\right\|_{\infty} \cdot|x-y|=0 .
$$

This implies the point-wise identity:

$$
f(x)-f(y)=\sum_{j \in \mathbb{Z}}\left(\left(P_{j} f\right)(x)-\left(P_{j} f\right)(y)\right) .
$$

Thus

$$
\begin{aligned}
|f(x)-f(y)| & \leq \sum_{j \leq J_{0}}\left\|\nabla P_{j} f\right\|_{\infty} \cdot|x-y|+\sum_{j>J_{0}} 2 \cdot\left\|P_{j} f\right\|_{\infty} \\
& \lesssim 2^{J_{0}(1-\alpha)}|x-y|\|f\|_{\dot{B}_{\infty, \infty}^{\alpha}}+2^{-J_{0} \alpha}\|f\|_{\dot{B}_{\infty, \infty}^{\alpha}} .
\end{aligned}
$$

Choosing $2^{J_{0}} \sim|x-y|^{-1}$ then yields the result.

Lemma 2.4. Let $0<\alpha<1$. Suppose $f \in \mathcal{S}^{\prime}\left(\mathbb{R}^{n}\right)$ satisfies

$$
\|f\|_{\dot{B}_{\infty, \infty}^{\alpha}}=\sup _{j}\left(\left\|P_{j} f\right\|_{\infty} \cdot 2^{j \alpha}\right)<\infty .
$$

Then

$$
f=\tilde{f}+p,
$$

where $p$ is a polynomial, and $\tilde{f}$ is a continuous function satisfying

$$
\|\tilde{f}\|_{\dot{C}^{\alpha}} \sim\|f\|_{\dot{B}_{\infty, \infty}^{\alpha}} .
$$


Proof. Denote $f_{J}=\sum_{-J \leq j \leq J} P_{j} f$. Clearly $\left\|f_{J}\right\|_{\dot{C}^{\alpha}} \lesssim\|f\|_{\dot{B}_{\infty, \infty}^{\alpha}}$. Set

$$
\tilde{f}_{J}(x)=f_{J}(x)-f_{J}(0) .
$$

Note that $\left|\tilde{f}_{J}(x)\right| \lesssim 1+|x|^{\alpha}$. By Arzelà-Ascoli one can extract a subsequence $\tilde{f}_{J_{k}}$ converging locally uniformly to a limit $\tilde{f}$. Clearly $\|\tilde{f}\|_{\dot{C}^{\alpha}} \lesssim\|f\|_{\dot{B}_{\infty, \infty}^{\alpha}}$. By the previous lemma we have $\|\tilde{f}\|_{\dot{C}^{\alpha}} \sim\|\tilde{f}\|_{\dot{B}_{\infty, \infty}^{\alpha}}$. Fix any $j_{0} \in \mathbb{Z}$. Clearly if $J$ is large we have $P_{j_{0}} \tilde{f}_{J}=P_{j_{0}} f$. By Lebesgue Dominated Convergence and sending $J_{k}$ to infinity we obtain $P_{j_{0}} \tilde{f}=P_{j_{0}} f$ for any $j_{0} \in \mathbb{Z}$. Thus $\tilde{f}$ and $f$ differ at most by a polynomial.

The following corollary records the usual fact that for $0<\alpha<1$ and $f \in \mathcal{S}^{\prime}\left(\mathbb{R}^{n}\right)$, one has the equivalence $\|f\|_{B_{\infty, \infty}^{\alpha}} \sim\|f\|_{C^{\alpha}}$.

Corollary 2.5. Let $0<\alpha<1$. Suppose $f$ is continuous and bounded on $\mathbb{R}^{n}$. Then

$$
\|f\|_{\dot{B}_{\infty, \infty}^{\alpha}} \sim\|f\|_{\dot{C}^{\alpha}} .
$$

Proof. If $\|f\|_{\dot{C}^{\alpha}}<\infty$, clearly then $\|f\|_{\dot{B}_{\infty, \infty}^{\alpha}} \lesssim\|f\|_{\dot{C}^{\alpha}}$. Conversely if $\|f\|_{\dot{B}_{\infty, \infty}^{\alpha}}<\infty$, then by the previous lemma, we have $f=\tilde{f}+p$, where $p$ is a polynomial, and $\|\tilde{f}\|_{\dot{C}^{\alpha}} \sim\|f\|_{\dot{B}_{\infty, \infty}^{\alpha}}$. Since $f$ is continuous and bounded, by considering the limit of the ratios $f(x) /|x|$ and $\tilde{f}(x) /|x|$ as $|x| \rightarrow \infty$, it follows easily that $p$ must be a constant. Thus $f=\tilde{f}+$ const from which the desired result follows.

Remark. Alternatively one can use the condition $\|f\|_{\infty}<\infty$ to obtain that for any $x \neq y$,

$$
\begin{aligned}
\lim _{J \rightarrow \infty}\left|P_{\leq-J} f(x)-P_{\leq-J} f(y)\right| & \lesssim \lim _{J \rightarrow \infty}\left\|\nabla P_{\leq-J} f\right\|_{\infty}|x-y| \\
& \lesssim \lim _{J \rightarrow \infty} 2^{-J}\|f\|_{\infty}|x-y|=0 .
\end{aligned}
$$

Repeating the proof in Lemma 2.3 then yields easily $\|f\|_{\dot{C}^{\alpha}} \lesssim\|f\|_{\dot{B}_{\infty, \infty}^{\alpha}}$.

We also need the following maximal function estimates.

Lemma 2.6 (Fefferman-Stein inequality, 2]). Let $f=\left(f_{j}\right)_{j=1}^{\infty}$ be a sequence of functions in $\mathbb{R}^{n}$. Let $1<q, r<\infty$. There exists a positive constant $A_{r, q, n}>0$ such that

$$
\left\|\left(\sum_{j=1}^{\infty}\left|\mathcal{M}_{1} f_{j}\right|^{r}\right)^{\frac{1}{r}}\right\|_{q} \leq A_{r, q, n}\left\|\left(\sum_{j=1}^{\infty}\left|f_{j}\right|^{r}\right)^{\frac{1}{r}}\right\|_{q} .
$$

Here $\mathcal{M}_{1} g$ is the maximal function defined by (here $|Q|$ denotes $n$-dimensional Lebesgue measure of $Q):\left(\mathcal{M}_{1} g\right)(x)=\sup _{Q} \frac{1}{|Q|} \int_{Q}|g(y)| d y$, where the sup is taken over all cubes $Q$ centered at $x$.

Remark 2.7. Of course the maximal operator $\mathcal{M}_{1}$ can be replaced by the usual uncentered maximal operator $\mathcal{M}$ on balls:

$$
(\mathcal{M} g)(x)=\sup _{B} \frac{1}{|B|} \int_{B}|g(y)| d y,
$$

where the sup is taken over all balls $B$ containing the point $x$.

As an immediate consequence of Lemma 2.6, we have the following estimate. 
Corollary 2.8. Let $\tilde{P}_{j}$ be a family of Littlewood-Paley type projection operators adapted to frequency $|\xi| \sim 2^{j}$, and obey the bound (often this is easy to check)

$$
\sup _{j \in \mathbb{Z}}\left|\left(\tilde{P}_{j} g\right)(x)\right| \leq \text { const } \cdot(\mathcal{M} g)(x), \quad \forall g .
$$

Then we have

$$
\left\|\left(\sum_{j}\left|\tilde{P}_{j} f_{j}\right|^{r}\right)^{\frac{1}{r}}\right\|_{q} \lesssim\left\|\left(\sum_{j}\left|f_{j}\right|^{r}\right)^{\frac{1}{r}}\right\|_{q}, \quad \forall 1<q, r<\infty .
$$

Several acceptable choices of $\tilde{P}_{j}$ include, for example, the fattened projection: $\tilde{P}_{j}=P_{j-1}+P_{j}+P_{j+1}$ and the projections involving derivatives: $\tilde{P}_{j}=2^{-j s}|\nabla|{ }^{s} P_{j}$, $s>0$. In later sections, we shall often use this inequality (mostly in the case $r=2$ ) without explicit mentioning. Note that alternatively one can prove (2.1) by using the usual vector-valued $\mathrm{CZ}$ operator theory.

Note that in (2.1), $q \in(1, \infty)$. Next we shall discuss an analogue of this estimate when $q \leq 1$. Such estimate will be used in dealing with estimates in Hardy spaces. And we start by a short review of Hardy spaces 1

We recall the definition of Hardy space $\mathcal{H}^{1}$ :

$$
\mathcal{H}^{1}\left(\mathbb{R}^{n}\right)=\left\{f \in L^{1}\left(\mathbb{R}^{n}\right): \quad \mathcal{R}_{j} f \in L^{1}, \quad \forall j=1, \cdots, n\right\},
$$

where $\mathcal{R}_{j}$ are the Riesz transforms $\left(\hat{R}_{j}(\xi)=-i \xi_{j} /|\xi|\right)$ on $\mathbb{R}^{n}$ (for $n=1$ it is just the Hilbert transform) with the norm

$$
\|f\|_{\mathcal{H}^{1}}=\|f\|_{1}+\sum_{j=1}^{n}\left\|\mathcal{R}_{j} f\right\|_{1} .
$$

We shall not need the atomic or maximal function characterization of $\mathcal{H}^{1}$. On the other hand, it is well-known that (see e.g. Chapter 6.4 on p37 of [3])

$$
\|f\|_{\mathcal{H}^{1}} \sim\|\mathcal{S} f\|_{1}
$$

where $\mathcal{S} f$ is the usual Littlewood-Paley square function $\mathcal{S} f=\left(\sum_{j \in \mathbb{Z}}\left|P_{j} f\right|^{2}\right)^{\frac{1}{2}}$. In view of this equivalence, we shall conveniently use the definition

$$
\|f\|_{\mathcal{H}^{1}}=\|\mathcal{S} f\|_{1}
$$

in this note without explicit mentioning.

Lemma 2.9. Suppose $u \in \mathcal{S}\left(\mathbb{R}^{n}\right)$ with $\operatorname{supp}(\hat{u}) \subset\{\xi:|\xi|<t\}$ for some $t>0$. Then for any $0<r<\infty$,

$$
\sup _{z \in \mathbb{R}^{n}} \frac{|u(x-z)|}{(1+t|z|)^{\frac{n}{r}}} \lesssim_{r, n}\left(\mathcal{M}\left(|u|^{r}\right)(x)\right)^{\frac{1}{r}} .
$$

Remark. To understand the meaning of the lemma one can take $x=0$ and $t=1$. Thanks to frequency localization, for given $z$ the value $u(z)$ is mainly controlled by the $L^{r}$ norm of $|u|$ in a ball of size $O(|z|)$ centered at the origin. The factor $|z|^{\frac{n}{r}}$ then provides the needed normalization for the maximal function.

Remark. See Lemma 2.3 of [4] for a proof under a much weaker condition that $u$ is a tempered distribution (i.e. one can replace $\mathcal{S}\left(\mathbb{R}^{n}\right)$ by $\mathcal{S}^{\prime}\left(\mathbb{R}^{n}\right)$ ).

\footnotetext{
${ }^{1}$ To keep it simple, we only discuss the estimates in Hardy space $\mathcal{H}^{1}$, the extension to general Hardy space $\mathcal{H}^{p}$ can be done without much effort.
} 
Proof. By scaling we may assume $t=1$. By using translation we only need to consider the case $x=0$. Assume first $1 \leq r<\infty$. Since $u=P_{<2} u$, we have

$$
u(z)=\int \psi(z-y) u(y) d y,
$$

where $\psi$ is the Schwartz function corresponding to $P_{<2}$. Clearly then

$$
\begin{aligned}
|u(z)| & \leq \int|\psi(z-y)|^{\frac{1}{r}}|u(y)||\psi(z-y)|^{1-\frac{1}{r}} d y \\
& \lesssim\left(\int|\psi(z-y)||u(y)|^{r} d y\right)^{\frac{1}{r}} \\
& \lesssim(1+|z|)^{\frac{n}{r}}\left(\mathcal{M}\left(|u|^{r}\right)(0)\right)^{\frac{1}{r}}
\end{aligned}
$$

where in the last step we used the simple inequality

$$
\int|\psi(z-y)||f(y)| d y \lesssim(1+|z|)^{n}(\mathcal{M} f)(0) .
$$

Now consider $0<r<1$. Clearly

$$
\begin{aligned}
|u(z)| & \leq \int|\psi(z-y)||u(y)|^{r}|u(y)|^{1-r} d y \\
& \leq \int|\psi(z-y)||u(y)|^{r}(1+|y|)^{\frac{n}{r}(1-r)} d y \sup _{x} \frac{|u(x)|^{1-r}}{(1+|x|)^{\frac{n}{r}(1-r)}} \\
& \lesssim(1+|z|)^{\frac{n}{r}} \mathcal{M}\left(|u|^{r}\right)(0) \cdot \sup _{x} \frac{|u(x)|^{1-r}}{(1+|x|)^{\frac{n}{r}(1-r)}} .
\end{aligned}
$$

This then easily leads to the desired inequality.

Corollary 2.10. Let $0<r<\infty$ and $c_{1}>0$. Then for any $j \in \mathbb{Z}$ and Schwartz $f$ with $\operatorname{supp}(\hat{f}) \subset\left\{\xi:|\xi|<c_{1} 2^{j}\right\}$, we have

$$
\left|\left(P_{\leq j} f\right)(x)\right| \lesssim_{r, c_{1}, n}\left(\mathcal{M}\left(|f|^{r}\right)(x)\right)^{\frac{1}{r}} .
$$

In the above estimate the operator $P_{\leq j}$ can be replaced by $P_{j}$ or any $\tilde{P}_{\leq j}, \tilde{P}_{j}$.

Proof. The case $1 \leq r<\infty$ is trivial. In particular it follows from the estimate $|f(x)| \lesssim \mathcal{M} f(x) \lesssim_{r}\left(\left(\mathcal{M}|f|^{r}\right)(x)\right)^{\frac{1}{r}}$ when $r>1$. Now consider $0<r<1$ and without loss of generality assume $j=1$. Clearly

$$
\begin{aligned}
\left|\left(P_{\leq 1} f\right)(x)\right| & \leq \int|\psi(y)||f(x-y)| d y \\
& \lesssim \int|\psi(y)|(1+|y|)^{\frac{n}{r}} \frac{|f(x-y)|}{(1+|y|)^{\frac{n}{r}}} d y \\
& \lesssim \sup _{y} \frac{|f(x-y)|}{(1+|y|)^{\frac{n}{r}}} \lesssim\left(\left(\mathcal{M}\left(|f|^{r}\right)\right)(x)\right)^{\frac{1}{r}},
\end{aligned}
$$

where in the last step we have used Lemma 2.9 (this is where the frequency localisation condition on $f$ is needed).

Remark. In Corollary 2.10, the frequency localisation condition $\left\{|\xi| \lesssim 2^{j}\right\}$ is not needed for $1 \leq r<\infty$. On the other hand, for $0<r<1$, this condition is indeed needed. For a simple counterexample, one can take (in 1D) $f(y)=\chi_{\delta<|y|<1}|y|^{-1}$ 
for which $\mathcal{M}\left(|f|^{r}\right)$ is finite. On the other hand, $\left(P_{\leq 1} f\right)(0)$ can be made arbitrarily large by taking $\delta \rightarrow 0+$.

Lemma 2.11. Let $0<q<\infty, 0<b<\infty$ and $c_{1}>0$. Let $\left\{f_{j}\right\}, j \in \mathbb{Z}$ be $a$ sequence of functions such that $\operatorname{supp}\left(\widehat{f}_{j}\right) \subset\left\{\xi:|\xi|<c_{1} 2^{j}\right\}$. Then

$$
\left\|\left(\sum_{j \in \mathbb{Z}}\left|\tilde{P}_{j} f_{j}\right|^{b}\right)^{\frac{1}{b}}\right\|_{L^{q}} \lesssim q, b, c_{1}, n\left\|\left(\sum_{j \in \mathbb{Z}}\left|f_{j}\right|^{b}\right)^{\frac{1}{b}}\right\|_{L^{q}} .
$$

Proof. Note that by Corollary 2.10,

$$
\left.\left|\left(\tilde{P}_{j} f_{j}\right)(x)\right| \lesssim\left(\mathcal{M}\left(\left|f_{j}\right|^{r}\right)\right)(x)\right)^{\frac{1}{r}}
$$

Then by taking $0<r<\min \{q, b\}$ and using Lemma 2.6. we get

$$
\begin{aligned}
\left\|\left(\tilde{P}_{j} f_{j}\right)_{l_{j}^{b}}\right\|_{L_{x}^{q}} & \lesssim\left\|\left(\left(\mathcal{M}\left(\left|f_{j}\right|^{r}\right)\right)^{\frac{1}{r}}\right)_{l_{j}^{b}}\right\|_{L_{x}^{q}} \\
& \lesssim\left\|\left(\mathcal{M}\left(\left|f_{j}\right|^{r}\right)\right)_{l_{j}^{\frac{b}{r}}}\right\|_{L_{x}^{\frac{q}{r}}}^{\frac{1}{r}} \\
& \lesssim\left\|\left(\left|f_{j}\right|^{r}\right)_{l_{j}^{\frac{b}{r}}}\right\|_{L_{x}^{\frac{1}{r}}} \lesssim\left\|\left(f_{j}\right)_{l_{j}^{b}}\right\|_{L_{x}^{q} .}
\end{aligned}
$$

We will need to use the following useful estimates sometimes without explicit mentioning.

Lemma 2.12. Let $1 \leq q, r \leq \infty$. If $s>0$, then

$$
\begin{aligned}
\left\|\left(2^{j s} P_{>j} f\right)_{l_{j}^{r}}\right\|_{L_{x}^{q}} & \lesssim\left\|\left(2^{j s} P_{j} f\right)_{l_{j}^{r}}\right\|_{L_{x}^{q}} ; \\
\left\|\left(2^{j s}\left\|P_{>j} f\right\|_{L_{x}^{q}}\right)\right\|_{l_{j}^{r}} & \lesssim\left\|\left(2^{j s}\left\|P_{j} f\right\|_{L_{x}^{q}}\right)\right\|_{l_{j}^{r}} .
\end{aligned}
$$

If $0<s<1$ and $1 \leq q<\infty$, then

$$
\left\|\left(2^{j(s-1)} \nabla P_{\leq j} f\right)_{l_{j}^{r}}\right\|_{L_{x}^{q}} \lesssim\left\|\left(2^{j s} P_{j} f\right) l_{j}^{r}\right\|_{L_{x}^{q}}
$$

If $0<s<1$ and $1 \leq q \leq \infty$, then

$$
\left\|\left(2^{j(s-1)}\left\|\nabla P_{\leq j} f\right\|_{L_{x}^{q}}\right)\right\|_{l_{j}^{r}} \lesssim\left\|\left(2^{j s}\left\|P_{j} f\right\|_{L_{x}^{q}}\right)\right\|_{l_{j}^{r}} .
$$

Proof. The inequality (2.3) follows from Young's inequality in $l^{r}$ space. For (2.4) one can first use triangle inequality in $L_{x}^{q}$ and then use Young's inequality in $l^{r}$ space to conclude.

For (2.5), just observe that by Young's inequality in $l^{r}$, we have

$$
\left(2^{j(s-1)} \nabla P_{\leq j} f\right)_{l_{j}^{r}} \lesssim\left(2^{j s} \tilde{P}_{j} P_{j} f\right)_{l_{j}^{r}},
$$

where $\tilde{P}_{j}$ has frequency localised to $\left\{\xi:|\xi| \sim 2^{j}\right\}$. The desired result then follows from Lemma 2.11 (here we need $q<\infty$ ).

The proof of (2.6) is simpler and therefore omitted. 


\section{Regularity of PRESSURE}

Proof of Theorem 1.1. Proof of (1.5). We first notice that the case $s=0$ follows from (1.3), the case $s=1$ follows from (1.4) and standard bounds for Riesz type operators. Therefore we may assume $0<s<1$ and estimate only the homogeneous $\dot{W}^{2 s, q}$-norm of $p$.

Denote $R_{l k}=(-\Delta)^{-1} \partial_{l} \partial_{k}$ and make the decomposition

$$
\begin{aligned}
p & =\sum_{l, k} \sum_{j}\left(R_{l k}\left(P_{\leq j-2} u_{l} P_{j} u_{k}\right)+R_{l k}\left(P_{j} u_{l} P_{\leq j-2} u_{k}\right)+R_{l k}\left(P_{j} u_{l} \tilde{P}_{j} u_{k}\right)\right) \\
& =:(A)+(B)+(C) .
\end{aligned}
$$

where $\tilde{P}_{j}=P_{j-1}+P_{j}+P_{j+1}$.

For the first piece, by using frequency localisation and the fact that

$$
\sum_{k} \partial_{k}\left(P_{\leq j-2} u_{l} P_{j} u_{k}\right)=\sum_{k}\left(\partial_{k} P_{\leq j-2} u_{l}\right) u_{k},
$$

we obtain

$$
\begin{aligned}
\left\||\nabla|^{2 s}(A)\right\|_{q} & \lesssim\left\|\left(2^{j(2 s-1)}\left|\nabla P_{\leq j-2} u\right| \cdot\left|P_{j} u\right|\right)_{l_{j}^{2}}\right\|_{q} \\
& \lesssim\left\|\left(2^{j(s-1)} \nabla P_{\leq j-2} u\right)_{l_{j}^{2}}\right\|_{2 q} \cdot\left\|\left(2^{j s} P_{j} u\right)_{l_{j}^{2}}\right\|_{2 q} \lesssim\|u\|_{\dot{W}^{s, 2 q}}^{2},
\end{aligned}
$$

where in the last step we have used Lemma 2.12

The estimate of (B) is similar to (A) and therefore omitted. For (C) we write $u_{l}$ and $u_{k}$ simply as $u$, and estimate

$$
\begin{aligned}
\left\||\nabla|^{2 s}(C)\right\|_{q} & \lesssim\left\|\left(2^{2 m s}\left|\sum_{j \geq m-10} P_{j} u \tilde{P}_{j} u\right|\right)_{l_{m}^{2}}\right\|_{q} \\
& \lesssim\left\|\left(2^{j s} P_{j} u\right)_{l_{j}^{2}}\right\|_{2 q}\left\|\left(2^{j s} \tilde{P}_{j} u\right)_{l_{j}^{2}}\right\|_{2 q} \lesssim\|u\|_{W^{s, 2 q}}^{2} .
\end{aligned}
$$

Proof of (1.6). Note here $0<s<1$. We use the same decomposition of $p$ as above. For the first piece, one has

$$
\begin{aligned}
\|(A)\|_{\dot{B}_{q, r}^{2 s}} & \lesssim\left\|\left(2^{j(2 s-1)}\left\|\nabla P_{\leq j-2} u\right\|_{2 q}\left\|P_{j} u\right\|_{2 q}\right)\right\|_{l_{j}^{r}} \\
& \lesssim\left\|\left(2^{j(s-1)}\left\|\nabla P_{\leq j-2} u\right\|_{2 q}\right)\right\|_{l_{j}^{2 r}} \cdot\|u\|_{\dot{B}_{2 q, 2 r}^{s}} \lesssim\|u\|_{\dot{B}_{2 q, 2 r}^{s}}^{2} .
\end{aligned}
$$

The estimate of (B) is identical and omitted. For (C) we have (here we only need to require $s>0$ )

$$
\|(C)\|_{\dot{B}_{q, r}^{2 s}} \lesssim\left\|\left(2^{2 m s}\left\|\sum_{j \geq m-10} P_{j} u \tilde{P}_{j} u\right\|_{q}\right)\right\|_{l_{m}^{r}} \lesssim\|u\|_{\dot{B}_{2 q, 2 r}^{s}}^{2} .
$$

Remark. The estimate (1.5) can be easily derived using the new fractional Leibniz rule introduced in [4. Namely by using Corollary 1.4 in [4] and taking $s_{1}=s_{2}=s$, $A_{l k}^{2 s}=|\nabla|^{2 s}(-\Delta)^{-1} \partial_{l} \partial_{k}$, and noting that $0<s<1$, we obtain

$$
\left\|A_{l k}^{2 s}\left(u_{l} u_{k}\right)-u_{k} A_{l k}^{2 s} u_{l}-u_{l} A_{l k}^{2 s} u_{k}\right\|_{\frac{q}{2}} \lesssim\left\||\nabla|^{s} u_{l}\right\|_{q}\left\||\nabla|^{s} u_{k}\right\|_{q} \lesssim\left\||\nabla|^{s} u\right\|_{q}^{2} .
$$

Summing in $l$ and $k$ (inside the norm) and using incompressibility then yields the result. 
Proof of Theorem 1.2. As was already mentioned before the case $0<s<1$ follows from (1.6) and the embedding of $\dot{B}_{1,1}^{0}$ into $\mathcal{H}^{1}$. Thus we only need to consider the case $s=1$. By using (3.1) and the same manipulations therein, we have

$$
\begin{aligned}
\left\||\nabla|^{2}(A)\right\|_{\mathcal{H}^{1}} & \lesssim\left\|\left(2^{j}\left|\nabla P_{\leq j-2} u\right| \cdot\left|P_{j} u\right|\right)_{l_{j}^{2}}\right\|_{1} \lesssim\left\|\left(\left|\nabla P_{\leq j-2} u\right|\right)_{l_{j}^{\infty}}\right\|_{2}\left\|\left(2^{j} P_{j} u\right)_{l_{j}^{2}}\right\|_{2} \\
& \lesssim\|\nabla u\|_{2}^{2} .
\end{aligned}
$$

For the third piece, a close inspection of the Besov case in Theorem 1.1 shows that

$$
\|(C)\|_{\dot{B}_{1,1}^{2}} \lesssim\|\nabla u\|_{2}^{2}
$$

Since $\left\||\nabla|^{2}(C)\right\|_{\mathcal{H}^{1}} \lesssim\left\||\nabla|^{2}(C)\right\|_{\dot{B}_{1,1}^{0}}$, the desired result follows easily.

Proof of Theorem 1.3. We first decompose the product $f \cdot g$ as

$$
f \cdot g=\underbrace{\sum_{j \in \mathbb{Z}} f_{\leq j-2} \cdot g_{j}}_{=:(a)}+\underbrace{\sum_{j \in \mathbb{Z}} f_{j} \cdot g_{\leq j-2}}_{=:(b)}+\underbrace{\sum_{j \in \mathbb{Z}} \tilde{f}_{j} \cdot g_{j}}_{=:(c)},
$$

where $\tilde{f}_{j}=P_{j-1} f+P_{j} f+P_{j+1} f$. We only need to estimate $(a)$ and $(c)$ since the estimate of $(b)$ is similar to $(a)$. For $(a)$, note that by frequency localisation, we have

$$
\begin{aligned}
\left\||\nabla|^{s}(a)\right\|_{\mathcal{H}^{1}} & \lesssim\left\|\left(2^{j s}\left|f_{\leq j-2}\right| \cdot\left|g_{j}\right|\right)_{l_{j}^{2}}\right\|_{1} \lesssim\left\|\left(2^{j s} g_{j}\right)_{l_{j}^{2}}\right\|_{p_{2}} \cdot\left\|\left(f_{\leq j-2}\right)_{l_{j}^{\infty}}\right\|_{p_{2}^{\prime}} \\
& \lesssim\left\||\nabla|^{s} g\right\|_{p_{2}} \cdot\|f\|_{p_{2}^{\prime}} .
\end{aligned}
$$

One should note that for this estimate we do not have severe constraints on the exponent $s$. On the other hand if $-1<s \leq 0$, then

$$
\left\||\nabla|^{s}(a)\right\|_{\mathcal{H}^{1}} \lesssim\left\|\left(2^{j s}\left|f_{\leq j-2}\right|\right)_{l_{j}^{\infty}}\right\|_{p_{1}}\left\|\left(g_{j}\right)_{l_{j}^{2}}\right\|_{p_{1}^{\prime}} \lesssim\left\||\nabla|^{s} f\right\|_{p_{1}}\|g\|_{p_{1}^{\prime}}
$$

Thus

$$
\left\||\nabla|^{s}(a)\right\|_{\mathcal{H}^{1}} \lesssim\left\{\begin{array}{l}
\left\||\nabla|^{s} g\right\|_{p_{2}} \cdot\|f\|_{p_{2}^{\prime}}, \quad \text { if } s>0 ; \\
\min \left\{\left|\left\||\nabla|^{s} f\right\|_{p_{1}}\|g\|_{p_{1}^{\prime}},\left\||\nabla|^{s} g\right\|_{p_{2}}\|f\|_{p_{2}^{\prime}}\right\}, \quad \text { if }-1<s \leq 0 .\right.
\end{array}\right.
$$

Now for the diagonal piece $(c)$, we first note that for $s>0$ the "Div-Curl" condition is not needed since

$$
\begin{aligned}
\left\||\nabla|^{s}(c)\right\|_{\mathcal{H}^{1}} & \lesssim\left\||\nabla|^{s}(c)\right\|_{\dot{B}_{1,1}^{0}} \\
& \lesssim \sum_{k} 2^{k s} \sum_{j \geq k-10}\left\|\tilde{f}_{j} g_{j}\right\|_{1} \\
& \lesssim \min \left\{\left\||\nabla|^{s} f\right\|_{p_{1}}\|g\|_{p_{1}^{\prime}},\left\||\nabla|^{s} g\right\|_{p_{2}}\|f\|_{p_{2}^{\prime}}\right\} .
\end{aligned}
$$

In the regime $-1<s \leq 0$ we need to exploit the "Div-Curl" condition. Since $\nabla \times g=0$ we can write $g=\nabla \psi$ for a scalar potential $\psi$. Since $\nabla \cdot f=0$, we have

$$
(c)=\sum_{j} \tilde{f}_{j} \cdot g_{j}=\sum_{j} \nabla \cdot\left(\tilde{f}_{j} \psi_{j}\right)
$$


Clearly by frequency localisation,

$$
\begin{aligned}
\left\||\nabla|^{s}(c)\right\|_{\mathcal{H}^{1}} & \lesssim\left\||\nabla|^{s}(c)\right\|_{\dot{B}_{1,1}^{0}} \lesssim\left\|\left(2^{k(s+1)} \sum_{j \geq k-10}\left|f_{j}\right| \cdot\left|\psi_{j}\right|\right)_{l_{k}^{1}}\right\|_{1} \\
& \lesssim\left\|\left(2^{j(s+1)}\left|f_{j}\right| \cdot\left|\psi_{j}\right|\right)_{l_{j}^{1}}\right\|_{1} \\
& \lesssim \min \left\{\left\||\nabla|^{s} f\right\|_{p_{1}}\|g\|_{p_{1}^{\prime}},\left\||\nabla|^{s} g\right\|_{p_{2}}\|f\|_{p_{2}^{\prime}}\right\} .
\end{aligned}
$$

Remark. We record here a quite useful (albeit simple) estimate extracted from the proof above. For any $\varphi \in C_{c}^{\infty}\left(\mathbb{R}^{n}\right), \psi \in C_{c}^{\infty}\left(\mathbb{R}^{n}\right)$ with support localised to an annulus (say $\{|\xi| \sim 1\}$ ), define

$$
\widehat{P_{j}^{\varphi}} f(\xi)=\varphi\left(2^{-j} \xi\right) \hat{f}(\xi), \quad \widehat{P_{j}^{\psi}} f(\xi)=\psi\left(2^{-j} \xi\right) \hat{f}(\xi) .
$$

Then for any $s>0$, any $s_{1}, s_{2} \geq 0$ with $s_{1}+s_{2}=s$, and any $f \in \mathcal{S}\left(\mathbb{R}^{n}\right), g \in \mathcal{S}\left(\mathbb{R}^{n}\right)$, we have

$$
\begin{aligned}
&\left\||\nabla|^{s}\left(\sum_{j} P_{j}^{\varphi} f P_{j}^{\psi} g\right)\right\|_{\mathcal{H}^{1}} \lesssim\left\|\sum_{j} P_{j}^{\varphi} f P_{j}^{\psi} g\right\|_{\dot{B}_{1,1}^{s}} \\
& \lesssim s, \varphi, \psi, n, r, s_{1}, s_{2} \\
&\left\||\nabla|^{s_{1}} f\right\|_{r}\left\||\nabla|^{s_{2}} g\right\|_{r^{\prime}}
\end{aligned}
$$

where $1<r<\infty, r^{\prime}=\frac{r}{r-1}$.

Similarly for any $s>0$, any $s_{1}, s_{2} \geq 0$ with $s_{1}+s_{2}=s$, any $1<p<\infty$, any $1<p_{1}, p_{2}<\infty$ with $\frac{1}{p}=\frac{1}{p_{1}}+\frac{1}{p_{2}}$, we have

$$
\left\||\nabla|^{s}\left(\sum_{j} P_{j}^{\varphi} f P_{j}^{\psi} g\right)\right\|_{p} \lesssim_{s, \varphi, \psi, n, p, p_{1}, p_{2}, s_{1}, s_{2}}\left\||\nabla|^{s_{1}} f\right\|_{p_{1}}\left\||\nabla|^{s_{2}} g\right\|_{p_{2}} .
$$

Also (the following corresponds to $p_{2}=\infty$ )

$$
\left\||\nabla|^{s}\left(\sum_{j} P_{j}^{\varphi} f P_{j}^{\psi} g\right)\right\|_{p} \lesssim_{s, \varphi, \psi, n, p, s_{1}, s_{2}}\left\||\nabla|^{s_{1}} f\right\|_{p}\left\||\nabla|^{s_{2}} g\right\|_{\dot{B}_{\infty, \infty}^{0}}
$$

Inspired by the "Div-Curl" theorem and the considerations above, we record below a simple fractional Leibniz rule in the frequency-localised context which is quite useful for practical purposes. For $s>0$, denote by $A^{s}$ a differential operator such that its symbol $\widehat{A^{s}}(\xi)$ is a homogeneous function of degree $s$ and $\widehat{A^{s}}(\xi) \in$ $C^{\infty}\left(\mathbb{S}^{n-1}\right)$ (for example: $\widehat{A^{s}}(\xi)=i|\xi|^{s-1} \xi_{1}$ which corresponds to $A^{s}=|\nabla|^{s-1} \partial_{1}$, or $\widehat{A^{s}}(\xi)=|\xi|^{s}$ which corresponds to $\left.A^{s}=|\nabla|^{s}=D^{s}\right)$. We have the following proposition.

Proposition 3.1. Let $s>0$. Then for any $1<p_{1}, p_{2}<\infty$ with $\frac{1}{p_{1}}+\frac{1}{p_{2}}=1$, any $s_{1}, s_{2} \geq 0$ with $s_{1}+s_{2}=s$, and any $f, g \in \mathcal{S}\left(\mathbb{R}^{n}\right)$, we have

$$
\begin{aligned}
\| A^{s}(f g) & -\sum_{j \in \mathbb{Z}} A^{s}\left(f_{\leq j-2} g_{j}\right)-\sum_{j \in \mathbb{Z}} A^{s}\left(f_{j} g_{\leq j-2}\right) \|_{\mathcal{H}^{1}} \\
& \lesssim\left\|A^{s}(f g)-\sum_{j \in \mathbb{Z}} A^{s}\left(f_{\leq j-2} g_{j}\right)-\sum_{j \in \mathbb{Z}} A^{s}\left(f_{j} g_{\leq j-2}\right)\right\|_{\dot{B}_{1,1}^{0}} \\
& \lesssim A^{s, s, s_{1}, s_{2}, p, p_{1}, p_{2}, n}\left\|D^{s_{1}} f\right\|_{p_{1}}\left\|D^{s_{2}} g\right\|_{p_{2}} .
\end{aligned}
$$


For any $1<p<\infty$, any $1<p_{1}, p_{2}<\infty$ with $\frac{1}{p_{1}}+\frac{1}{p_{2}}=\frac{1}{p}$, any $s_{1}, s_{2} \geq 0$ with $s_{1}+s_{2}=s$, and any $f, g \in \mathcal{S}\left(\mathbb{R}^{n}\right)$, we have

$$
\begin{aligned}
\| A^{s}(f g) & -\sum_{j \in \mathbb{Z}} A^{s}\left(f_{\leq j-2} g_{j}\right)-\sum_{j \in \mathbb{Z}} A^{s}\left(f_{j} g_{\leq j-2}\right) \|_{p} \\
& \lesssim A^{s, s, s_{1}, s_{2}, p, p_{1}, p_{2}, n}\left\|D^{s_{1}} f\right\|_{p_{1}}\left\|D^{s_{2}} g\right\|_{p_{2}} .
\end{aligned}
$$

Also

$$
\begin{gathered}
\left\|A^{s}(f g)-\sum_{j \in \mathbb{Z}} A^{s}\left(f_{\leq j-2} g_{j}\right)-\sum_{j \in \mathbb{Z}} A^{s}\left(f_{j} g_{\leq j-2}\right)\right\|_{p} \\
\lesssim A^{s, s, s_{1}, s_{2}, p, n}\left\|D^{s_{1}} f\right\|_{p}\left\|D^{s_{2}} g\right\|_{\dot{B}_{\infty, \infty}^{0}} .
\end{gathered}
$$

Remark. Since $f, g \in \mathcal{S}\left(\mathbb{R}^{n}\right)$, one does not need to worry about the convergence issues. For example, it is easy to check that $\sum_{j} f_{\leq j-2} g_{j} \in H^{m}\left(\mathbb{R}^{n}\right), \sum_{j} \tilde{f}_{j} g_{j} \in$ $W^{m, 2}\left(\mathbb{R}^{n}\right)$, for all $m \geq 0$, and

$$
\begin{aligned}
& A^{s}\left(\sum_{j} f_{\leq j-2} g_{j}\right)=\sum_{j} A^{s}\left(f_{\leq j-2} g_{j}\right), \\
& A^{s}\left(\sum_{j} \tilde{f}_{j} g_{j}\right)=\sum_{j} A^{s}\left(\tilde{f}_{j} g_{j}\right),
\end{aligned}
$$

where the above equality holds point-wisely for $x \in \mathbb{R}^{n}$ and in stronger norms.

Proof of Proposition 3.1. This follows from the para-product decomposition, (3.2), (3.3) and (3.4).

Corollary 3.2. Let $s>0$. For any $1<r_{1}, r_{2}<\infty$, we have

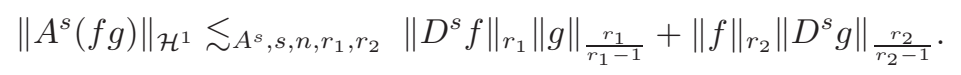

For any $1<p<\infty$, any $1<p_{1}, p_{3}<\infty, 1<p_{2}, p_{4} \leq \infty$ with $\frac{1}{p}=\frac{1}{p_{1}}+\frac{1}{p_{2}}=\frac{1}{p_{3}}+\frac{1}{p_{4}}$, we have

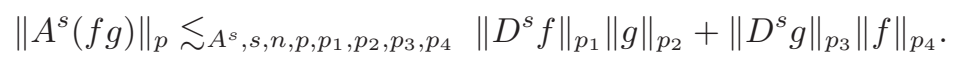

Proof. This follows easily from Proposition 3.1 For example, one observes that

$$
\left\|\sum_{j} A^{s}\left(f_{\leq j-2} g_{j}\right)\right\|_{p} \lesssim\left\|\left(2^{j s} g_{j}\right)_{l_{j}^{2}}\right\|_{p_{3}}\left\|\left(f_{\leq j-2}\right)_{l_{j}^{\infty}}\right\|_{p_{4}} \lesssim\left\|D^{s} g\right\|_{p_{3}}\|f\|_{p_{4}} .
$$

The other cases are similarly estimated. We omit the details.

\section{COUNTEREXAMPles}

Proof of Theorem 1.4, case $s=1$. Step 1: Some simplification. We shall take $u=$ $\left(u_{1}, u_{2}, 0,0, \cdots, 0\right)$, where

$$
u_{1}=-\partial_{2} \phi, \quad u_{2}=\partial_{1} \phi,
$$

and $\phi: \mathbb{R}^{n} \rightarrow \mathbb{R}$ will be chosen later. Note that by construction $u$ is divergence-free. Now

$$
\begin{aligned}
-\Delta p & =\sum_{j, k} \partial_{j} u_{k} \partial_{k} u_{j} \\
& =\left(\partial_{1} u_{1}\right)^{2}+\left(\partial_{2} u_{2}\right)^{2}+2 \partial_{1} u_{2} \partial_{2} u_{1} \\
& =2\left(\left(\partial_{12} \phi\right)^{2}-\partial_{11} \phi \partial_{22} \phi\right)
\end{aligned}
$$


Clearly, the task now is to find a scalar function $\phi \in \mathcal{S}\left(\mathbb{R}^{n}\right)$ with $\|\phi\|_{2}+$ $\|\phi\|_{\dot{B}_{\infty, \infty}^{2}} \leq 1$, such that

$$
\left\|\left(\partial_{12} \phi\right)^{2}-\partial_{11} \phi \partial_{22} \phi\right\|_{\dot{B}_{\infty, \infty}^{0}} \gg 1 .
$$

As a matter of fact, we shall show that for some $j_{1} \gg 1$,

$$
\left\|P_{j_{1}}\left(\left(\partial_{12} \phi\right)^{2}-\partial_{11} \phi \partial_{22} \phi\right)\right\|_{\infty} \gg 1
$$

i.e. the norm inflation occurs at a high frequency block.

Step 2: There exists $\phi_{0} \in \mathcal{S}\left(\mathbb{R}^{n}\right)$ with $\operatorname{supp}\left(\widehat{\phi_{0}}\right) \subset\left\{\xi: \frac{2}{3} \leq|\xi| \leq \frac{5}{6}\right\}$ and

$$
\left(\left(\partial_{12} \phi_{0}\right)^{2}-\partial_{11} \phi_{0} \partial_{22} \phi_{0}\right)(0) \neq 0 \text {. }
$$

Indeed one can take $\widehat{\phi_{0}}(\xi)$ as a radial real-valued function in $\xi$ with

$$
\begin{gathered}
\int \xi_{1} \xi_{2} \widehat{\phi_{0}}(\xi) d \xi=0, \\
\int \xi_{1}^{2} \widehat{\phi_{0}}(\xi) d \xi \neq 0, \\
\int \xi_{2}^{2} \widehat{\phi_{0}}(\xi) d \xi \neq 0 .
\end{gathered}
$$

These yield $\left(\partial_{12} \phi_{0}\right)(0)=0,\left(\partial_{11} \phi_{0}\right)(0) \cdot\left(\partial_{22} \phi_{0}\right)(0) \neq 0$.

Step 3: Now take $J \gg 1, J$ being an even integer and

$$
\phi(x)=\sum_{j=100}^{J} 2^{-2 j^{2}} \phi_{0}\left(2^{j^{2}} x\right) .
$$

Easy to check that (note that $\left.\operatorname{supp}\left(\hat{\phi}_{0}\right) \subset\left\{\frac{2}{3} \leq|\xi| \leq \frac{5}{6}\right\} \subset\left\{\frac{1}{2}<|\xi|<\frac{7}{6}\right\}\right)$

$$
\|\phi\|_{2}+\|\phi\|_{\infty}+\|\phi\|_{\dot{B}_{\infty, \infty}^{2}} \lesssim 1
$$

Then clearly

$$
\begin{aligned}
& \left(\partial_{12} \phi(x)\right)^{2} \\
= & \sum_{j=100}^{J}\left(\partial_{12} \phi_{0}\right)\left(2^{j^{2}} x\right)\left(\partial_{12} \phi_{0}\right)\left(2^{j^{2}} x\right) \\
& +2 \sum_{j_{1}=100}^{J}\left(\partial_{12} \phi_{0}\right)\left(2^{j_{1}^{2}} x\right)\left(\sum_{100 \leq j_{2}<j_{1}}\left(\partial_{12} \phi_{0}\right)\left(2^{j_{2}^{2}} x\right)\right) .
\end{aligned}
$$

Similarly

$$
\begin{aligned}
& \partial_{11} \phi(x) \partial_{22} \phi(x) \\
= & \sum_{j=100}^{J}\left(\partial_{11} \phi_{0}\right)\left(2^{j^{2}} x\right)\left(\partial_{22} \phi_{0}\right)\left(2^{j^{2}} x\right) \\
& +\sum_{j_{1}=100}^{J}\left(\partial_{11} \phi_{0}\right)\left(2^{j_{1}^{2}} x\right)\left(\sum_{100 \leq j_{2}<j_{1}}\left(\partial_{22} \phi_{0}\right)\left(2^{j_{2}^{2}} x\right)\right) \\
& +\sum_{j_{1}=100}^{J}\left(\partial_{22} \phi_{0}\right)\left(2^{j_{1}^{2}} x\right)\left(\sum_{100 \leq j_{2}<j_{1}}\left(\partial_{11} \phi_{0}\right)\left(2^{j_{2}^{2}} x\right)\right) .
\end{aligned}
$$


Thus

$$
\begin{aligned}
& \left(\partial_{12} \phi(x)\right)^{2}-\partial_{11} \phi(x) \partial_{22} \phi(x) \\
= & H_{1}(x)+H_{2}(x)
\end{aligned}
$$

where

$$
H_{1}(x)=\sum_{j=100}^{J}\left(\left(\partial_{12} \phi_{0}\right)^{2}-\partial_{11} \phi_{0} \partial_{22} \phi_{0}\right)\left(2^{j^{2}} x\right),
$$

is the "diagonal piece", and $H_{2}(x)$ is the off-diagonal piece.

We shall show

$$
\left\|P_{\leq 1} H_{1}\right\|_{\infty}+\left\|H_{1}\right\|_{\dot{B}_{\infty, \infty}^{0}} \lesssim 1, \quad\left\|P_{j_{1}} H_{2}\right\|_{\infty} \gg 1, \quad \text { for some } j_{1} \gg 1 .
$$

Step 4: the diagonal piece. One can rewrite $H_{1}$ as

$$
\begin{aligned}
2 H_{1}(x) & =\partial_{1}\left(\sum_{j=100}^{J} 2^{-j^{2}}\left(\partial_{2} \phi_{0} \partial_{12} \phi_{0}-\partial_{1} \phi_{0} \partial_{22} \phi_{0}\right)\left(2^{j^{2}} x\right)\right) \\
& +\partial_{2}\left(\sum_{j=100}^{J} 2^{-j^{2}}\left(-\partial_{2} \phi_{0} \partial_{11} \phi_{0}+\partial_{1} \phi_{0} \partial_{12} \phi_{0}\right)\left(2^{j^{2}} x\right)\right) .
\end{aligned}
$$

Thanks to the above expression, it is easy to check that

$$
\left\|P_{\leq 1} H_{1}\right\|_{\infty} \lesssim \sum_{j \geq 100} 2^{-j^{2}} \lesssim 1 .
$$

Note that on the Fourier side, we have roughly speaking

$$
\widehat{H_{1}}(\xi) \sim \xi \sum_{j=100}^{J} c_{j} 1_{|\xi| \leq \frac{5}{3} 2^{j^{2}}}
$$

It is then easy to check that for each $l \geq 2$ (note that $P_{l}$ has frequency localised to $\left.\frac{1}{2} 2^{l} \leq|\xi| \leq \frac{7}{6} 2^{l}\right)$,

$$
\left\|P_{l} H_{1}\right\|_{\infty} \lesssim 2^{l} \sum_{j \geq \sqrt{l-2}} 2^{-j^{2}} \lesssim 1
$$

Step 5: The off-diagonal piece. Take $j_{0}=J / 2$ and $j_{1}=j_{0}^{2}$. It is not difficult to check that

$$
\begin{aligned}
P_{j_{1}} H_{2}= & 2\left(\partial_{12} \phi_{0}\right)\left(2^{j_{0}^{2}} x\right) \sum_{100 \leq j_{2}<j_{0}}\left(\partial_{12} \phi_{0}\right)\left(2^{j_{2}^{2}} x\right) \\
& -\left(\partial_{11} \phi_{0}\right)\left(2^{j_{0}^{2}} x\right) \sum_{100 \leq j_{2}<j_{0}}\left(\partial_{22} \phi_{0}\right)\left(2^{j_{2}^{2}} x\right) \\
& -\left(\partial_{22} \phi_{0}\right)\left(2^{j_{0}^{2}} x\right) \sum_{100 \leq j_{2}<j_{0}}\left(\partial_{11} \phi_{0}\right)\left(2^{j_{2}^{2}} x\right) .
\end{aligned}
$$

Thus

$$
\left|\left(P_{j_{1}} H_{2}\right)(0)\right| \gtrsim j_{0}\left|\left(\partial_{12} \phi_{0}\right)(0)^{2}-\left(\partial_{11} \phi_{0}\right)(0)\left(\partial_{22} \phi_{0}\right)(0)\right| \gg 1 .
$$

This yields the desired result. 
Proof of Theorem 1.4, case $s=0$. We shall take $u=\left(-\partial_{2} \phi, \partial_{1} \phi, 0, \cdots, 0\right)$ where $\phi: \mathbb{R}^{n} \rightarrow \mathbb{R}$ will be specified momentarily.

Step 1. First we choose Schwartz $g: \mathbb{R}^{n} \rightarrow \mathbb{R}$ with $\operatorname{supp}(\hat{g}) \subset\left\{\xi: \frac{1}{2} \leq|\xi| \leq 2\right\}$ such that

$$
\left(P_{j_{0}} \Delta^{-1} \partial_{11}\left(g^{2}\right)\right)(0) \neq 0, \quad \text { for any } j_{0} \in \mathbb{Z} .
$$

This can be easily achieved since $\Delta^{-1} \partial_{11}$ corresponds to the symbol $\xi_{1}^{2} /|\xi|^{2}$ and one can just take $\hat{g}$ to be non-negative.

Step 2. Let $J \gg 1$ and define

$$
\phi(x)=\sum_{j=1}^{J} \frac{1}{k_{j}} \cos \left(k_{j} x_{2}\right) g(x),
$$

where $k_{j}$ are dyadic numbers which are well separated and will be chosen sufficiently large. Note that $\|\phi\|_{\dot{B}_{\infty, \infty}^{1}}+\|\phi\|_{2} \lesssim 1$.

Step 3. Non-diagonal term does not matter. Note that $\|u\|_{\dot{B}_{\infty, \infty}^{0}} \lesssim 1$. Recall

$$
-p=\sum_{l, k} \Delta^{-1} \partial_{l} \partial_{k}\left(u_{l} u_{k}\right) .
$$

Clearly

$$
\begin{aligned}
& \left\|\sum_{l, k} \Delta^{-1} \partial_{l} \partial_{k}\left(\sum_{j} P_{\leq j-2} u_{l} P_{j} u_{k}\right)\right\|_{\dot{B}_{\infty, \infty}^{0}} \\
\lesssim & \sup _{j} \sum_{l, k}\left\|\Delta^{-1} \partial_{l}\left(\partial_{k} P_{\leq j-2} u_{l} P_{j} u_{k}\right)\right\|_{\infty} \lesssim\|u\|_{\dot{B}_{\infty, \infty}^{0}}^{2} \lesssim 1 .
\end{aligned}
$$

Step 4. Inflation through the diagonal terms. In terms of $\phi$, we have

$$
-p=\Delta^{-1} \partial_{11}\left(\left(\partial_{2} \phi\right)^{2}\right)+\Delta^{-1} \partial_{22}\left(\left(\partial_{1} \phi\right)^{2}\right)-2 \Delta^{-1} \partial_{12}\left(\partial_{1} \phi \partial_{2} \phi\right) .
$$

Clearly

$$
\partial_{2} \phi=\sum_{j=1}^{J}\left(-\sin \left(k_{j} x_{2}\right) g(x)+\frac{1}{k_{j}} \cos \left(k_{j} x_{2}\right) \partial_{2} g\right) .
$$

Then

$$
\begin{aligned}
\left(\partial_{2} \phi\right)^{2}= & \text { "Non-diagonal terms" } \\
& +\sum_{j=1}^{J}\left(\sin ^{2}\left(k_{j} x_{2}\right) g(x)^{2}+\frac{1}{k_{j}^{2}} \cos ^{2}\left(k_{j} x_{2}\right)\left(\partial_{2} g\right)^{2}\right) \\
= & \text { "Non-diagonal terms" } \\
& +\frac{1}{2} J g(x)^{2}+\frac{1}{2}\left(\sum_{j=1}^{J} \frac{1}{k_{j}^{2}}\right)\left(\partial_{2} g\right)^{2} \\
& +\frac{1}{2} \sum_{j=1}^{J}\left(-\cos \left(2 k_{j} x_{2}\right) g(x)^{2}+\frac{1}{k_{j}^{2}} \cos \left(2 k_{j} x_{2}\right)\left(\partial_{2} g\right)^{2}\right) .
\end{aligned}
$$

It follows that

$$
\left\|-p-\frac{J}{2} \Delta^{-1} \partial_{11}\left(g^{2}\right)\right\|_{\dot{B}_{\infty, \infty}^{0}} \lesssim 1 .
$$


Thus for any $j_{0} \in \mathbb{Z}$, by taking $J$ large, we obtain

$$
\left|\left(P_{j_{0}} p\right)(0)\right| \gtrsim \text { const } \cdot J \gg 1 \text {. }
$$

This clearly implies the desired inflation of $\|p\|_{\dot{B}_{\infty, \infty}^{0}}$ in high frequency.

We now consider the case $u \in \dot{C}^{\frac{1}{2}}=\dot{B}_{\infty, \infty}^{\frac{1}{2}}$.

Proposition 4.1. For any $\epsilon>0$, there exists divergence free $u \in \mathcal{S}\left(\mathbb{R}^{n}\right)$ with $\|u\|_{\dot{B}_{\infty}^{\frac{1}{2}, \infty}}+\|u\|_{2} \leq 1$ such that

$$
\|\nabla p\|_{\infty}>\frac{1}{\epsilon}
$$

Proof of Proposition 4.1 .

Step 1: Take a scalar function $\phi_{0} \in \mathcal{S}\left(\mathbb{R}^{n}\right)$ with $\operatorname{supp}\left(\widehat{\phi_{0}}\right) \subset\left\{\xi: \frac{2}{3} \leq|\xi| \leq \frac{5}{6}\right\}$. Let $J \gg 1$ and

$$
\phi(x)=\sum_{j=1000}^{J} N_{j}^{-\frac{3}{2}} \phi_{0}\left(N_{j} x\right),
$$

where $N_{j}=2^{a_{j}}, a_{j}=3^{j^{2}}$. Note that by construction we have

$$
N_{j-1}<N_{j}^{\frac{1}{2}}, \quad \forall j .
$$

Denote $\nabla^{\perp}=\left(-\partial_{2}, \partial_{1}, 0, \cdots, 0\right)$. Then define

$$
u=\nabla^{\perp} \phi=\sum_{j=1000}^{J} \underbrace{N_{j}^{-\frac{1}{2}}\left(\nabla^{\perp} \phi_{0}\right)\left(N_{j} x\right)}_{=: v_{j}} .
$$

Easy to check that

$$
\|u\|_{\dot{B}_{\infty, \infty}^{\frac{1}{2}}}+\|u\|_{2} \lesssim 1
$$

On the other hand,

$$
\begin{aligned}
\nabla p= & (-\Delta)^{-1} \nabla \nabla \cdot((u \cdot \nabla) u) \\
= & \sum_{j}(-\Delta)^{-1} \nabla \nabla \cdot\left(\left(v_{j} \cdot \nabla\right) v_{j}\right) \\
& +\sum_{j}(-\Delta)^{-1} \nabla \nabla \cdot\left(\left(v_{j} \cdot \nabla\right) v_{\leq j-1}\right) \\
& +\sum_{j}(-\Delta)^{-1} \nabla \nabla \cdot\left(\left(v_{\leq j-1} \cdot \nabla\right) v_{j}\right),
\end{aligned}
$$

where we have denoted $v_{\leq j-1}=\sum_{i \leq j-1} v_{i}$.

By (4.7), frequency localization and Bernstein, we have

$$
\|(4.9)\|_{\infty} \lesssim \sum_{j} N_{j}^{-\frac{1}{2}} N_{j-1}^{\frac{1}{2}} \lesssim 1
$$


For (4.10), we shall transform it to a similar form as in (4.9). Note that for any two vector functions $f, g$ with $\nabla \cdot f=\nabla \cdot g=0$, we have

$$
\begin{aligned}
\nabla \cdot((f \cdot \nabla) g) & =\sum_{k, l} \partial_{k} f_{l} \partial_{l} g_{k} \\
& =\sum_{l} \partial_{l}\left(\sum_{k}\left(\partial_{k} f_{l}\right) g_{k}\right) .
\end{aligned}
$$

Thanks to the above transformation, it is clear that we have

$$
\nabla \cdot\left(\left(v_{\leq j-1} \cdot \nabla\right) v_{j}\right)=O\left(\partial\left(\partial v_{\leq j-1} v_{j}\right)\right)
$$

Thus it can be estimated in the same way as in (4.9), and we have

$$
\|(4.10)\|_{\infty} \lesssim_{j} N_{j}^{-\frac{1}{2}} N_{j-1}^{\frac{1}{2}} \lesssim 1 \text {. }
$$

Step 2: From step 1, it is clear that the dominant contribution to $\|\nabla p\|_{\infty}$ comes from the diagonal piece (4.8). Denote (4.8) as $H(x)$. Then by using scaling, we have

$$
\begin{aligned}
H(0) & =\sum_{j}\left((-\Delta)^{-1} \nabla \nabla \cdot\left(\left(v_{j} \cdot \nabla\right) v_{j}\right)\right)(0) \\
& =(J-999)\left((-\Delta)^{-1} \nabla \nabla \cdot\left(\left(\nabla^{\perp} \phi_{0}\right) \cdot \nabla\right)\left(\nabla^{\perp} \phi_{0}\right)\right)(0) .
\end{aligned}
$$

Thus we only need to show the existence of $\phi_{0}$ such that

$$
\left|\left((-\Delta)^{-1} \nabla \nabla \cdot\left(\left(\nabla^{\perp} \phi_{0}\right) \cdot \nabla\right)\left(\nabla^{\perp} \phi_{0}\right)\right)(0)\right| \neq 0 .
$$

This is not difficult to do and we sketch the detail below.

First by using the proof of Theorem 1.4 (see Step 2 therein), one can find $\tilde{\phi}_{0} \in$ $\mathcal{S}\left(\mathbb{R}^{n}\right)$ with $\operatorname{supp}\left(\widehat{\tilde{\phi}_{0}}\right) \subset\left\{\xi: \frac{2}{3} \leq|\xi| \leq \frac{5}{6}\right\}$ such that

$$
\begin{aligned}
& \left(\nabla \cdot\left(\left(\nabla^{\perp} \tilde{\phi}_{0} \cdot \nabla\right)\left(\nabla^{\perp} \tilde{\phi}_{0}\right)\right)\right)(0) \\
= & 2\left(\left(\partial_{12} \tilde{\phi}_{0}\right)^{2}-\partial_{11} \tilde{\phi}_{0} \partial_{22} \tilde{\phi}_{0}\right)(0) \neq 0 .
\end{aligned}
$$

Take a smooth bump function $g$ such that

$$
\int_{\mathbb{R}^{n}}\left(\nabla \cdot\left(\left(\nabla^{\perp} \tilde{\phi}_{0} \cdot \nabla\right)\left(\nabla^{\perp} \tilde{\phi}_{0}\right)\right)\right)(x) g(x) d x \neq 0 .
$$

Clearly then

$$
\int_{\mathbb{R}^{n}}\left((-\Delta)^{-1} \nabla \nabla \cdot\left(\left(\nabla^{\perp} \tilde{\phi}_{0} \cdot \nabla\right)\left(\nabla^{\perp} \tilde{\phi}_{0}\right)\right)\right)(x) \cdot \nabla g(x) d x \neq 0 .
$$

From this, one sees that there exists $x_{*}$ such that

$$
\left((-\Delta)^{-1} \nabla \nabla \cdot\left(\left(\nabla^{\perp} \tilde{\phi}_{0} \cdot \nabla\right)\left(\nabla^{\perp} \tilde{\phi}_{0}\right)\right)\right)\left(x_{*}\right) \neq 0 .
$$

Define $\phi_{0}(x)=\tilde{\phi}_{0}\left(x+x_{*}\right)$. Since translation in physical space is equivalent to modulation in frequency space (which does not change frequency localisation), it is not difficult to check that $\phi_{0}$ satisfies all the required properties.

The next proposition requires a slightly different construction, in the sense that the frequency supports are no longer disjoint. We only need to work in the physical space. 
Proposition 4.2. For any $\epsilon>0$, there exists divergence free $u \in \mathcal{S}\left(\mathbb{R}^{n}\right)$ with $\|\nabla u\|_{\infty}+\|u\|_{2} \leq 1$ such that

$$
\|\nabla p\|_{C^{1}}>\frac{1}{\epsilon}
$$

Proof of Proposition 4.2. Take a smooth bump function $\phi_{0} \in C_{c}^{\infty}\left(\mathbb{R}^{n}\right)$ with

$$
\operatorname{supp}\left(\phi_{0}\right) \subset\left\{x: c_{1}<|x|<c_{2}\right\}
$$

for some constants $0<c_{1}<c_{2}<\infty$. Define

$$
\phi(x)=\sum_{j=1}^{J} k_{j}^{-2} \phi_{0}\left(k_{j} x\right),
$$

where $J \gg 1$, and $k_{j}$ are dyadic numbers sufficiently large such that each summand has disjoint support. Denote $\nabla^{\perp}=\left(-\partial_{2}, \partial_{1}, 0, \cdots, 0\right)$ and

$$
u=\nabla^{\perp} \phi=\sum_{j=1}^{J} \underbrace{k_{j}^{-1}\left(\nabla^{\perp} \phi_{0}\right)\left(k_{j} x\right)}_{=: v_{j}} .
$$

Obviously $\|u\|_{C^{1}}+\|u\|_{2} \lesssim 1$ (since the rescaled copies of $\nabla^{\perp} \phi_{0}$ have disjoint supports). On the other hand, by using scaling,

$$
\begin{aligned}
\left(\partial_{11} p\right)(0) & =\left((-\Delta)^{-1} \partial_{11} \nabla \cdot((u \cdot \nabla) u)\right)(0) \\
& =\sum_{j=1}^{J}\left((-\Delta)^{-1} \partial_{11} \nabla \cdot\left(v_{j} \cdot \nabla v_{j}\right)\right)(0) \\
& =J\left((-\Delta)^{-1} \partial_{11} \nabla \cdot\left(\left(\nabla^{\perp} \phi_{0} \cdot \nabla\right) \nabla^{\perp} \phi_{0}\right)\right)(0) .
\end{aligned}
$$

Thus we only need to choose $\phi_{0}$ compactly supported on an annulus such that

$$
\left|\left((-\Delta)^{-1} \partial_{11} \nabla \cdot\left(\left(\nabla^{\perp} \phi_{0} \cdot \nabla\right) \nabla^{\perp} \phi_{0}\right)\right)(0)\right| \neq 0 .
$$

This is not difficult to achieve and we sketch the details below.

Step 1. Simplification. We shall choose $\phi_{0}$ to have support away from the origin. This way one does not need to worry about the singular kernels in the integration by part argument below. Denote $v=\nabla^{\perp} \phi_{0}$. Then

$$
\begin{aligned}
& \left(\Delta^{-1} \partial_{11} \nabla \cdot(v \cdot \nabla v)\right)(0) \\
= & \sum_{1 \leq j, k \leq 2} \int v_{j} v_{k} \partial_{j} \partial_{k}(\underbrace{\partial_{11} \Delta^{-1} \delta_{0}}_{=: a(x)}) d x \\
= & \int\left(v_{1}^{2} \partial_{11} a+v_{2}^{2} \partial_{22} a-2 v_{1} v_{2} \partial_{12} a\right) d x .
\end{aligned}
$$

Step 2. Case dimension $n=2$. Note that

$$
a(x)=C_{1} \cdot\left(\left(x_{1}^{2}+x_{2}^{2}\right)^{-1}-2\left(x_{1}^{2}+x_{2}^{2}\right)^{-2} x_{1}^{2}\right),
$$

where $C_{1}>0$ is an absolute constant. Denote $\tilde{a}(x)=a(x) / C_{1}$. Easy to verify that

$$
\left(\partial_{11} \tilde{a}\right)(1,0)=-6, \quad\left(\partial_{22} \tilde{a}\right)(1,0)=6, \quad\left(\partial_{12} \tilde{a}\right)(1,0)=0 .
$$


Now define $x_{*}=(1,0)$ and let

$$
\phi_{0}(x)=\cos \left(k x_{2}\right) c\left(\frac{x-x_{*}}{\eta}\right),
$$

where $c$ is a bump function localised to the unit ball, $\eta>0$ will be taken sufficiently small (so that $\left|\left(\partial_{11} \tilde{a}\right)(x)\right| \sim 1$ for $\left|x-x_{*}\right|<\eta$ ) and $k$ will be taken sufficiently large. It is then easy to check (recall $v_{1}=-\partial_{2} \phi_{0}$ )

$$
\begin{aligned}
&\left|\int\left(v_{1}^{2} \partial_{11} \tilde{a}+v_{2}^{2} \partial_{22} \tilde{a}-2 v_{1} v_{2} \partial_{12} \tilde{a}\right) d x\right| \\
& \geq \text { const } \cdot k^{2}-O(k) \gtrsim 1,
\end{aligned}
$$

if $k$ is sufficiently large. This settles the $2 \mathrm{D}$ case.

Step 3. Case dimension $n \geq 3$. Denote $x_{*}=(1,0, \cdots, 0)$. Easy to check that

$$
\left.\partial_{11} a(x)\right|_{x=x_{*}}=-\left.\operatorname{const} \cdot \partial_{x_{1}}^{4}\left(x_{1}^{-(n-2)}\right)\right|_{x_{1}=1} \neq 0
$$

Again let

$$
\phi_{0}(x)=\cos \left(k x_{2}\right) c\left(\frac{x-x_{*}}{\eta}\right)
$$

will yield

$$
\begin{aligned}
& \quad\left|\int\left(v_{1}^{2} \partial_{11} \tilde{a}+v_{2}^{2} \partial_{22} \tilde{a}-2 v_{1} v_{2} \partial_{12} \tilde{a}\right) d x\right| \\
& \geq \mathrm{const} \cdot k^{2}-O(k) \gtrsim 1 .
\end{aligned}
$$

This settles the case for $n \geq 3$.

Proof of Proposition 1.6. Define $\nabla^{\perp}=\left(-\partial_{2}, \partial_{1}, 0, \cdots, 0\right)$. Let $\phi \in C_{c}^{\infty}\left(\mathbb{R}^{n}\right)$ be such that

$$
\begin{aligned}
& \int_{\mathbb{R}^{n}}\left(\partial_{1} \phi\right)^{2} d x \neq \int_{\mathbb{R}^{n}}\left(\partial_{2} \phi\right)^{2} d x, \\
& \int_{\mathbb{R}^{n}} \partial_{1} \phi \partial_{2} \phi d x=0 .
\end{aligned}
$$

For example, one can take $\phi(x)=b(x) \cos \left(k x_{1}\right)$, where $b \in C_{c}^{\infty}\left(\mathbb{R}^{n}\right)$ is radial, and $k$ is sufficiently large.

Define $u=\nabla^{\perp} \phi$. By using a computation similar to that in (4.1), we have

$$
p=2(-\Delta)^{-1}(\underbrace{\left(\partial_{12} \phi\right)^{2}-\partial_{11} \phi \partial_{22} \phi}_{:=g(x)}) .
$$

Easy to check that $\hat{g}(0)=0$ and $\left(\partial_{\xi} \hat{g}\right)(0)=0$. The latter is due to the fact that

$$
\int_{\mathbb{R}^{n}}\left(\left(\partial_{12} \phi\right)^{2}-\partial_{11} \phi \partial_{22} \phi\right) x_{j} d x=0, \quad \forall 1 \leq j \leq n .
$$

To derive this, one can use the simple identity (below $\beta=\beta(x)$ denotes a smooth weight function)

$$
\begin{aligned}
\int \partial_{11} \phi \partial_{22} \phi \beta(x) d x=\int & \left(\partial_{12} \phi\right)^{2} \beta \\
& +\frac{1}{2} \int\left(-\left(\partial_{2} \phi\right)^{2} \partial_{11} \beta-\left(\partial_{1} \phi\right)^{2} \partial_{22} \beta+2 \partial_{1} \phi \partial_{2} \phi \partial_{12} \beta\right) d x
\end{aligned}
$$


On the other hand, by using the identity above, it is not difficult to check that

$$
\begin{aligned}
& \int_{\mathbb{R}^{n}} g(x) x_{1}^{2} d x=\int_{\mathbb{R}^{n}}\left(\partial_{2} \phi\right)^{2} d x, \\
& \int_{\mathbb{R}^{n}} g(x) x_{2}^{2} d x=\int_{\mathbb{R}^{n}}\left(\partial_{1} \phi\right)^{2} d x, \\
& \int_{\mathbb{R}^{n}} g(x) x_{1} x_{2} d x=-\int_{\mathbb{R}^{n}} \partial_{1} \phi \partial_{2} \phi d x=0 .
\end{aligned}
$$

Thus near $\xi=0$,

$$
\hat{g}(\xi)=c_{1} \xi_{1}^{2}+c_{2} \xi_{2}^{2}+O\left(|\xi|^{4}\right),
$$

where $c_{1} \neq c_{2}$. Clearly then $\hat{g}(\xi) /|\xi|^{2}$ is not continuous at $\xi=0$. This immediately implies that $p \notin L^{1}\left(\mathbb{R}^{n}\right)$.

\section{REFERENCES}

[1] R. P. Coifman, P. L. Lions, Y. Meyer, and S. Semmes. Compensated compactness and Hardy spaces. J. Math. Pures. Appl. 72(1992), 247-286.

[2] C. Fefferman and E. M. Stein. Some maximal inequalities. Amer. J. Math. 93(1971), 107-116.

[3] L. Grafakos. Modern Fourier analysis. 2ed. Springer 2009.

[4] D. Li. On Kato-ponce and fractional Leibniz. To appear in Revista Matemática Iberoamericana.

Department of Mathematics, The Hong Kong University of Science and Technology, Clear Water Bay, Kowloon, Hong Kong

Email address: madli@ust.hk

Department of Mathematics, University of Iowa, 14 Maclean Hall, Iowa City, IA 52242

Email address: xiaoyi-zhang@uiowa.edu 